\title{
USPIO-enhanced MRI of pelvic lymph nodes at 7-T: preliminary experience
}

\author{
Bart W. J. Philips ${ }^{1}$ (1) - Rutger C. H. Stijns ${ }^{1} \cdot$ Stefan H. G. Rietsch ${ }^{2,3} \cdot$ Sascha Brunheim $^{2,3} \cdot$ Jelle O. Barentsz ${ }^{1}$. \\ Ansje S. Fortuin ${ }^{1,4} \cdot$ Harald H. Quick ${ }^{2,3}$. Stephan Orzada ${ }^{2,3} \cdot$ Marnix C. Maas $^{1} \cdot$ Tom W. J. Scheenen ${ }^{1,2}$
}

Received: 5 July 2018 / Revised: 16 April 2019 / Accepted: 17 May 2019 / Published online: 14 June 2019

(C) The Author(s) 2019

\begin{abstract}
Purpose To evaluate the technical feasibility of high-resolution USPIO-enhanced magnetic resonance imaging of pelvic lymph nodes (LNs) at ultrahigh magnetic field strength.

Materials and methods The ethics review board approved this study and written informed consent was obtained from all patients. Three patients with rectal cancer and three selected patients with (recurrent) prostate cancer were examined at 7-T 24-36 h after intravenous ferumoxtran-10 administration; rectal cancer patients also received a 3-T MRI. Pelvic LN imaging was performed using the TIAMO technique in combination with water-selective multi-GRE imaging and lipidselective GRE imaging with a spatial resolution of $0.66 \times 0.66 \times 0.66 \mathrm{~mm}^{3} . \mathrm{T}_{2}{ }^{*}$-weighted images of the water-selective imaging were computed from the multi-GRE images at $\mathrm{TE}=0,8$, and $14 \mathrm{~ms}$ and used for the assessment of USPIO uptake.

Results High-resolution 7-T MR gradient-echo imaging was obtained robustly in all patients without suffering from RF-related signal voids. USPIO signal decay in LNs was visualized using computed TE imaging at $\mathrm{TE}=8 \mathrm{~ms}$ and an $\mathrm{R}_{2}{ }^{*}$ map derived from water-selective imaging. Anatomically, LNs were identified on a combined reading of computed TE $=0 \mathrm{~ms}$ images from waterselective scans and images from lipid-selective scans. A range of 3-48 LNs without USPIO signal decay was found per patient. These LNs showed high signal intensity on computed TE $=8$ and 14 ms imaging and low $\mathrm{R}_{2}{ }^{*}$ (corresponding to high $\mathrm{T}_{2}{ }^{*}$ ) values on the $\mathrm{R}_{2}^{*}$ map.

Conclusion USPIO-enhanced MRI of the pelvis at 7-T is technically feasible and offers opportunities for detecting USPIO uptake in normal-sized LNs, due to its high intrinsic signal-to-noise ratio and spatial resolution.

Key Points

- USPIO-enhanced MRI at 7-T can indicate USPIO uptake in lymph nodes based on computed TE images.

- Our method promises a high spatial resolution for pelvic lymph node imaging.
\end{abstract}

Keywords Ferumoxtran-10 $\cdot$ Lymph nodes $\cdot$ Pelvis $\cdot$ Metastasis $\cdot$ Magnetic resonance imaging

Bart W. J. Philips

bart.philips@ radboudumc.nl

1 Department of Radiology and Nuclear Medicine (766), Radboud University Medical Center, P.O. Box 9101,

Nijmegen, The Netherlands

2 Erwin L Hahn Institute for Magnetic Resonance Imaging, University Duisburg-Essen, 45141 Essen, Germany

3 High-Field and Hybrid MR Imaging, University Hospital Essen, 45147 Essen, Germany

4 Department of Radiology, Ziekenhuis Gelderse Vallei, Ede, The Netherlands

\begin{tabular}{|c|c|}
\hline \multicolumn{2}{|c|}{ Abbreviations } \\
\hline B1TIAMO & $\begin{array}{l}\mathrm{B}_{1}^{+} \text {mapping technique for TIAMO } \mathrm{B}_{1}^{+} \text {shim } \\
\text { calculation }\end{array}$ \\
\hline FA & Flip angle \\
\hline FOV & Field of view \\
\hline GRE & Gradient echo \\
\hline PLND & Pelvic lymph node dissection \\
\hline $\mathrm{RF}$ & Radiofrequency \\
\hline ROI & Region of interest \\
\hline RSS & Root sum of squares \\
\hline RSS FA & Root sum of squares flip angle \\
\hline SNR & Signal-to-noise ratio \\
\hline $\mathrm{TE}$ & Echo time \\
\hline
\end{tabular}




\begin{tabular}{|c|c|}
\hline TIAMO & Time interleaved acquisition of modes \\
\hline TME & Total mesorectal excision \\
\hline USPIO & $\begin{array}{l}\text { Ultrasmall superparamagnetic particles of iron } \\
\text { oxide }\end{array}$ \\
\hline WLLS & Weighted linear least squares \\
\hline
\end{tabular}

\section{Introduction}

Lymph nodes often are the first organs to which cancer metastasizes. It is important to detect metastatic nodes at an early stage as a signal of systematic disease, such that adequate treatment can be provided. Prostate cancer, rectal cancer, and gynecological cancers, the major cancers that occur within the pelvis, all metastasize primarily to pelvic lymph nodes [1] and their lymph node status in patients is still primarily diagnosed using invasive surgical techniques, such as a pelvic lymph node dissection [2].

Given its high sensitivity (82-96\%) and specificity (9398\%) [3-7], MRI in combination with a lymphotropic ultrasmall superparamagnetic particles of iron oxide (USPIO) contrast agent may be a valid non-invasive alternative for pelvic lymph node staging and has recently gained renewed interest $[8,9]$. In short, intravenously administered USPIOs accumulate in healthy lymph nodes, strongly attenuating the MR signal in $\mathrm{T}_{2}{ }^{*}$-weighted imaging. Suspicious lymph nodes without USPIO uptake retain their MR signal. Although the reported sensitivities and specificities of this technique are promising, they depend on study design, inclusion criteria, and reader experience $[5,10,11]$. Furthermore, the sensitivity decreases to $41 \%$ for lymph nodes smaller than $5 \mathrm{~mm}$ [4]. These studies were performed on 1.5-T MR systems in the early 2000s [4]. Later work $[11,12]$ already showed improved image quality at $3-\mathrm{T}$ versus $1.5-\mathrm{T}$, indicating that higher field strengths can improve the detection of lymph node metastases, particularly for lymph nodes smaller than $5 \mathrm{~mm}$ [13-15]. Moreover, a higher spatial resolution may allow a better identification of lymph node resembling structures such as nerve ganglia.

New technologies such as 7-T MRI systems offer a substantial increase in intrinsic signal-to-noise ratio (SNR) [16]. Moreover, the decrease of $\mathrm{T}_{2}{ }^{*}$ in non-metastatic lymph nodes due to USPIO uptake is more pronounced at higher field strengths due to increased susceptibility effects [17]. These effects can be used to further enhance spatial resolution and increase the signal difference between USPIO accumulating normal lymph nodes and metastatic lymph nodes retaining MR signal $[18,19]$.

MR imaging of the pelvis at 7-T is technically challenging due to severe signal voids that are caused by RF transmission interferences [20]. Time-interleaved acquisition of modes (TIAMO) [21] is a method that efficiently uses two different shim settings to acquire two complementary images that are combined into one image with an apparent homogeneous RF excitation. This method has been used in combination with water- and lipid-selective excitation to obtain high-resolution 7-T MR images of the pelvis in healthy volunteers [19]. Because imaging was performed with a low flip angle, $\mathrm{T}_{2}{ }^{*}$ sensitive gradient echo (GRE) sequence, this method can easily be adapted for USPIO-enhanced 7-T MR imaging without any issues with increased radiofrequency power deposition.

The aim of this paper is to demonstrate the technical feasibility of 7-T high-resolution USPIO-enhanced MRI of pelvic lymph nodes in patients with pelvic cancers.

\section{Methods}

\section{Subjects}

To illustrate the technical feasibility of our method, three patients with histologically proven prostate cancer were selected based on the presence of multiple small lymph nodes without USPIO uptake on their clinical 3-T examination. These patients were asked permission to perform a 7-T examination. Three additional patients with histologically proven rectal cancer were included before undergoing a total mesorectal excision and underwent 3-T as well as 7-T examinations (part of a larger patient trial of 20 patients, clinical trials identifier NCT02751606). The study was approved by the ethics review board (CMO region Arnhem-Nijmegen and the Ethics committee of the Medical Faculty of the University of DuisburgEssen) and signed informed consent was obtained for inclusion into the study from all patients before participation. Patients were excluded when contra-indications for 7-T MRI (metal implants, epilepsy, abdominal circumference $>120 \mathrm{~cm}$ ) or USPIO contrast (any prior contrast reaction, allergy to dextran or ferumoxtran-10, hemochromatosis, thalassemia, sickle cell anemia) were present and all inclusions were performed prospectively between June 2014 and October 2017. All patients (see Table 1 for clinical information) were scanned 24-36 h after an intravenous infusion of USPIOs (ferumoxtran-10, which is an investigational product produced by Radboudumc; from September 2015: SPL Medical B.V.) [9] of 2.6-mg iron per kg of bodyweight. For the infusion, the USPIOs were diluted in $100 \mathrm{cc}$ saline solution and administered over $30 \mathrm{~min}$ using a Minisart NML $0.22-\mu \mathrm{m}$ pore size filter (Minisart NML Syringe Filters 16,534-k; Sartorius AG). Patients were observed during and shortly after the 30-min contrast administration period for adverse reactions. On the day of the MRI scan, patients were asked whether any adverse reactions had occurred. All rectal cancer patients were scanned before surgical treatment. All patients received intramuscular smooth muscle and bowel relaxant and rectal cancer patients received a laxative (Table 2). 


\section{Measurement setup}

All 7-T MRI measurements were performed on a whole-body research MR system (MAGNETOM 7-T, Siemens Healthcare $\mathrm{GmbH})$. The patients with prostate cancer were examined using a custom-built 8 -channel transceiver ( $8 \mathrm{Tx} / 8 \mathrm{Rx})$ bodyarray coil [22] and the three patients with rectal cancer were scanned using a custom-built combined 8-channel transmit / 32-channel receive $(8 \mathrm{Tx} / 32 \mathrm{Rx})$ body array [23], both with meander-type microstrip elements [24]. TIAMO shimming was performed in all subjects, using a phase and amplitude RF shimming algorithm to optimize two complementary $\mathrm{B}_{1}{ }^{+}$ modes for root-sum-of-squares (RSS) homogeneity [25]. For measurements with the $8 \mathrm{Tx} / 8 \mathrm{Rx}$ coil, $\mathrm{B}_{1}{ }^{+}$-shimming was based on a series of single-slice low flip angle (FA) GRE images of the individual coil elements with the slice positioned transversally through the pelvis. The $8 \mathrm{Tx} / 32 \mathrm{Rx}$ measurements were performed using the $\mathrm{B} 1 \mathrm{TIAMO} \mathrm{B}_{1}{ }^{+}$mapping technique [26], which, next to relative $\mathrm{B}_{1}{ }^{+}$maps, also yielded absolute $\mathrm{B}_{1}^{+}$results.

The 3-T MRI scan (MAGNETOM Prisma-Fit, Siemens Healthcare $\mathrm{GmbH}$ ) in patients with rectal cancer was performed with table and body-phased array coils (Table 2).

\section{Imaging}

After a localizer, $\mathrm{B}_{0}$ and $\mathrm{B}_{1}{ }^{+}$shimming, a large field of view (FOV) 3D slab-selective GRE image was acquired for an anatomical overview (FOV $384 \times 384 \times 192 \mathrm{~mm}^{3}$, matrix $384 \times$ $384 \times 192$, repetition time (TR) $4.1 \mathrm{~ms}$, echo time (TE) $1.81 \mathrm{~ms}$, RSS FA of $6^{\circ}$ over 2 TRs, acquisition time 1:33 min). For water-selective imaging, five echoes were acquired (referred to as "original images") using a multigradient echo (mGRE) sequence. A monoexponential decay model was fitted to the signal decay across the five echoes for every pixel in these images using a weighted linear least squares (WLLS) algorithm [19, 27], yielding maps of the $\mathrm{R}_{2}{ }^{*}$ relaxation rate (where $\mathrm{R}_{2}{ }^{*}=1 / T_{2}{ }^{*}$ ). The WLLS weights the measurement points based on their signal level to account for the non-zero mean of noise in magnitude images. Based on the fit results, computed echo time images (referred to as "computed TE images") were reconstructed at TEs 0,8 , and $14 \mathrm{~ms}$. In addition, a lipid-selective imaging series was performed for an additional complementary anatomical overview of the lower abdomen at the same spatial resolution. The FOV covered at least the area from the aortic bifurcation to the bottom of the urinary bladder (for prostate imaging) and rectum (for rectal imaging). The sequence parameters are summarized in Table 2.

Both 3-T and 7-T scans were performed within the 24-36-h timeframe after USPIO contrast administration, with no more than $5 \mathrm{~h}$ between the start of the studies. 


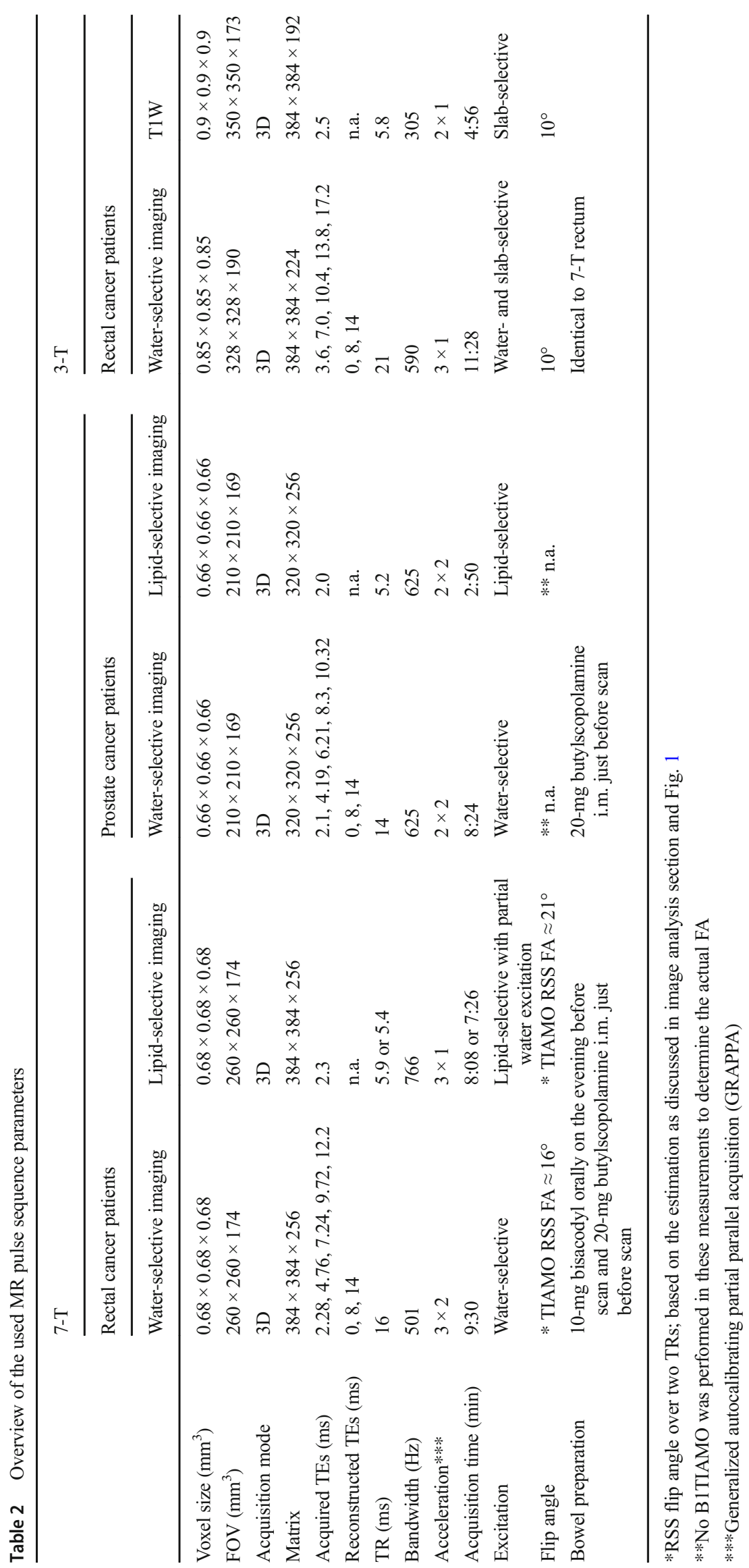




\section{Image analysis}

As RF transmit inhomogeneity leading to inhomogeneous FA distributions is a major issue in 7-T body imaging, FA maps were acquired with the B1TIAMO technique to evaluate the individual FA distributions. We calculated the effective FA distribution (mean \pm standard deviation (SD)) in the waterand lipid-selective imaging by determining the FA range within a region of interest (ROI) relevant for lymph node imaging.

To evaluate the $\mathrm{T}_{2}{ }^{*}$ signal decay within lymph nodes, ROIs were drawn within the lymph nodes on the original $\mathrm{TE}=$ $2.1 \mathrm{~ms}$ images and copied to the original images at the other TEs. A WLLS fit of the voxel-averaged signal at different TEs within these ROIs was performed. To account for the Rician noise distribution of magnitude images, the average intensity of the noise of a nearby ROI within lipid tissue (no signal on the water-selective images) was subtracted from each lymph node ROI signal. The uncertainty of the $\mathrm{R}_{2}{ }^{*}$ value in each node was calculated based on the fitting accuracy and expressed as a standard deviation.

To provide initial descriptive findings of visible lymph nodes in the six patients, the images were evaluated by one of two radiologists ( 7 and 11 years of experience) to assess the USPIO signal decay of lymph nodes on the isotropic 3D datasets in at least two orientations. The lymph nodes were identified on a combined reading of lipid-selective images and computed TE $=0 \mathrm{~ms}$ images of water-selective scans. Nodes were annotated as "without USPIO signal decay" if they retained high signal intensity on the computed TE $=8 \mathrm{~ms}$ imaging according to a "metastatic" or "possibly metastatic" pattern as described in Heesakkers et al [5]. The short axis of lymph nodes without USPIO signal decay was evaluated on the computed $\mathrm{TE}=8 \mathrm{~ms}$ image by the coordinating researcher (B.W.J. Philips).

\section{Results}

No adverse drug reactions occurred during contrast administration and all patients accepted the 7-T MRI scan without any complaints. We performed susceptibility-sensitive water- and lipid-selective pelvic imaging at 7-T without suffering from any RF-related signal voids in any of the patients, using the TIAMO technique (Fig. 1). Total measurement time, including patient and RF coil positioning and calibration measurements, was less than $1 \mathrm{~h}$. Only at the superior and inferior edges of the large FOV images, some reduced signal was observed due to the limited size of the RF coil array in this dimension. Within the depicted ROI (Fig. 1c), the calculated RSS FA over two TRs was $16.2 \pm 2.8^{\circ}$ (mean \pm SD) for the water-selective imaging and $21.0 \pm 3.6^{\circ}$ for the lipid-selective imaging.

Reading the combination of lipid series and water-selective $\mathrm{TE}=0 \mathrm{~ms}$ images, lymph nodes with high and low USPIO signal decay could be detected. A range of 3-48 lymph nodes without USPIO signal decay were found in the six patients with a maximum short-axis diameter within the range of 1.5$8.5 \mathrm{~mm}$ (Table 1). Normal lymph nodes, which show high USPIO uptake, appeared dark on the water-selective computed TE imaging at long TE due to the strong $\mathrm{R}_{2}{ }^{*}$ decay effectuated by the USPIOs. This resulted in high $\mathrm{R}_{2}{ }^{*}$ values on the $\mathrm{R}_{2}{ }^{*}$ maps. Suspicious lymph nodes, without USPIO signal decay, had a high signal intensity at long $\mathrm{TE}$ and low $\mathrm{R}_{2}{ }^{*}$ values, as they did not experience the strong $\mathrm{R}_{2}{ }^{*}$ effect of
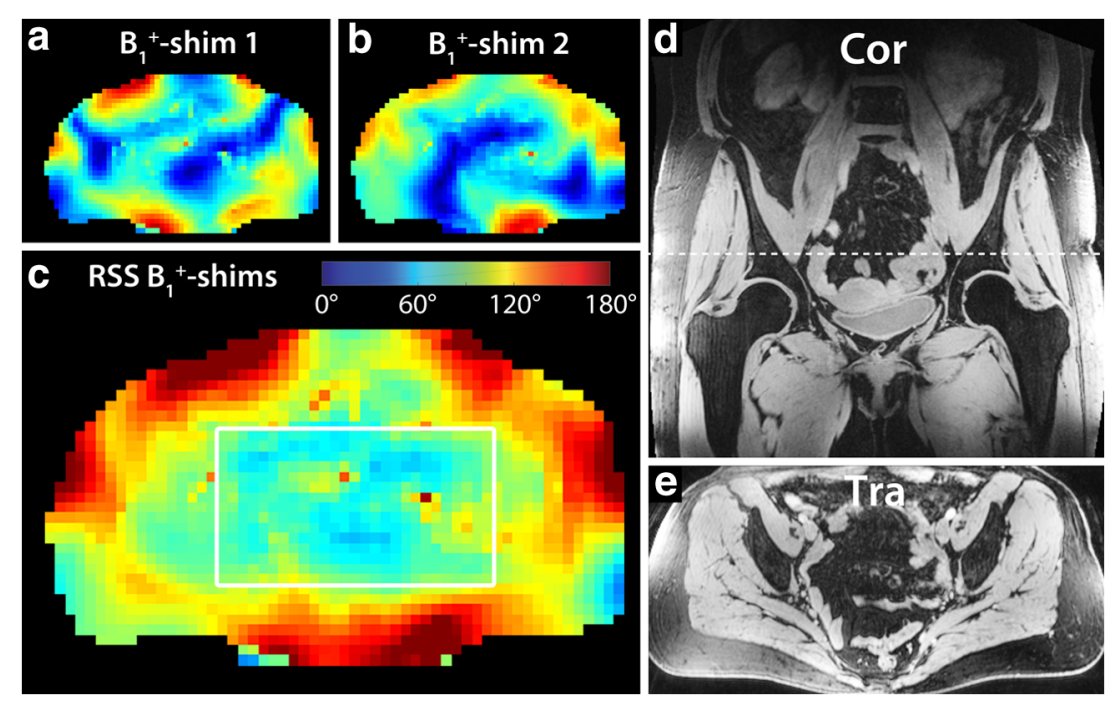

Fig. 1 The TIAMO flip angle maps corresponding to the two individual $\mathrm{B}_{1}{ }^{+}$shim settings (a and $\mathbf{b}$ ) and the combined root sum of squares (RSS) flip angle map (c) in patient 5 (male, 64 years old, rectal cancer). The white box in $\mathbf{c}$ indicates the ROI in which the flip angle distribution was evaluated. The two complementary shim settings produced a RSS homogenous flip angle distribution over a large FOV (water-selective GRE overview image example in $\mathbf{d}$ and e) (see also Table 2). A single axial slice (a-c) was used for $\mathrm{B}_{1}{ }^{+}$shimming (indicated by dashed line in coronal image d). The corresponding axial overview GRE image is depicted in $\mathbf{e}$ 
USPIOs. With extrapolation to TE $=0 \mathrm{~ms}$, the signal intensity of lymph nodes with high USPIO uptake was recovered, such that computed TE imaging was also used for detecting normal lymph nodes (Fig. 2). Computed TE images had a higher SNR than the original images, but similar contrast (Fig. 2b, e). Lymph nodes that take up USPIO contrast only within a small part of the cortex could also be observed, as illustrated by the 4.5-mm lymph node shown in Fig. $2 \mathrm{i}-\mathrm{k}$, which shows distinct regions of high and low USPIO signal decay.
Water-selective 7-T lymph node imaging was performed at a higher spatial resolution in a similar acquisition time compared with 3-T. In the three patients with rectal cancer examined on 3-T and 7-T, vessel and node delineation were sharper at 7-T (example in Fig. 3). The high spatial resolution at 7-T enabled visualization of lymph nodes down to sizes of $1.5 \mathrm{~mm}$ short axis (Fig. 4). In the water-selective images at 3-T, the blood vessels were somewhat hyperintense due to the T1shortening effect of USPIO contrast in the blood pool.
Fig. 2 Imaging at 7-T of patient 1 (male, 54 years old, prostate cancer). A sagittal image is shown at different computed TEs $(\mathbf{a}-\mathbf{c})$, lipid-selective imaging (d) and the original mGRE waterselective imaging at TE $8.3 \mathrm{~ms}$ (e). In addition, a map of $\mathrm{R}_{2}{ }^{*}$ relaxation rates obtained from the WLLS fit is depicted (f). Three lymph nodes with USPIO signal decay (white circles) and one lymph node without USPIO signal decay (white arrow) were annotated. A sagittal overview image is also shown (g). The GRE signal decay of a normal LN with USPIO signal decay (annotated in images a-f with asterisk) and a suspicious LN with low USPIO signal decay (annotated with number sign) are depicted in $\mathbf{h}$. The lymph node marked with a number sign showed a slow signal decay with a low $R_{2}{ }^{*}$ value of 80 $\pm 6 \mathrm{~s}^{-1}$, whereas the lymph node marked with an asterisk showed a fast $\mathrm{R}_{2}{ }^{*}$ decay with a high $\mathrm{R}_{2}{ }^{*}$ value of $247 \pm 25 \mathrm{~s}^{-1}$. In addition, a lymph node is shown with homogenous high signal intensity at the computed $\mathrm{TE}=0 \mathrm{~ms}$ image (i) that shows partial USPIO signal decay in a small region, indicated by the arrow. In this region, signal intensity on the TE $=8 \mathrm{~ms}$ image (j) is low and the $\mathrm{R}_{2}{ }^{*}$ values on the $\mathrm{R}_{2}{ }^{*}$ map (k) are high, whereas the rest of the lymph node shows low USPIO signal decay indicated by high signal intensity at $\mathrm{TE}=8 \mathrm{~ms}$ and low $\mathrm{R}_{2}{ }^{*}$ values
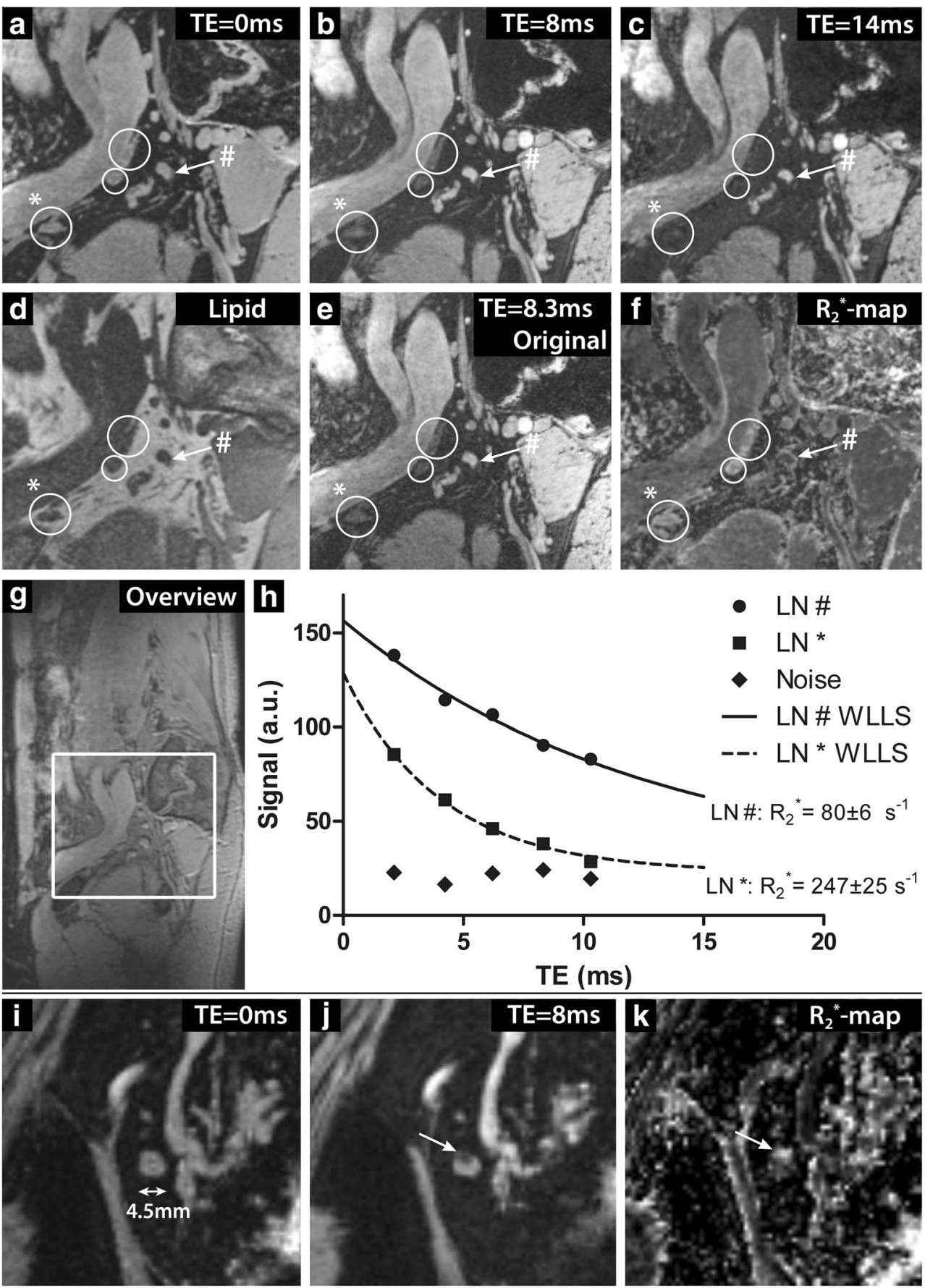

- LN \#

- $\mathrm{LN}$ *

- Noise

- LN \# WLLS

-.. LN * WLLS
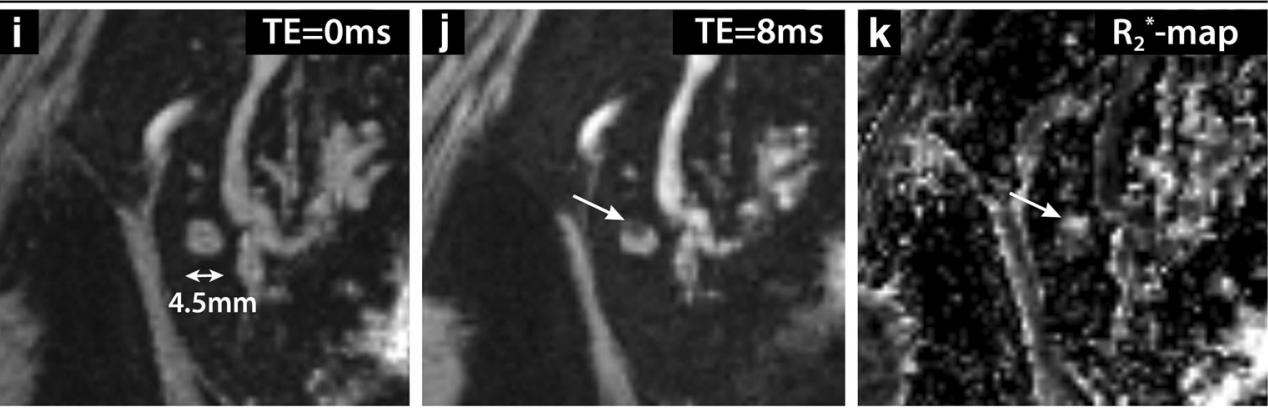

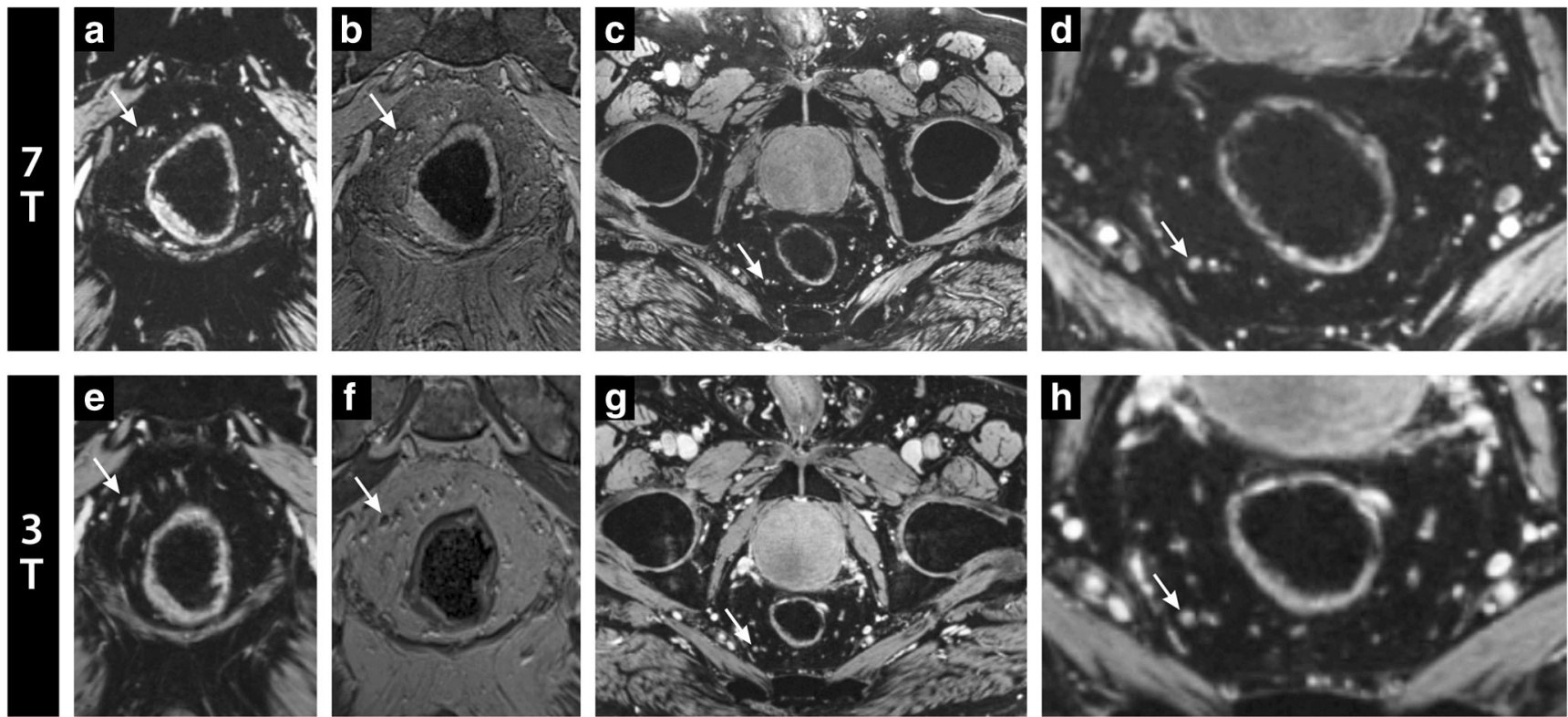

Fig. 3 Water- (computed TE $=8 \mathrm{~ms})(\mathbf{a}, \mathbf{c}-\mathbf{e}, \mathbf{g}$, and $\mathbf{h})$ and lipid-selective imaging (b and f) at 7-T (top) and 3-T (bottom) in coronal (a, b, e, and f) and axial direction (c, d, g, and $\mathbf{h}$ ) of patient 4 (male, 77 years old, rectal cancer). A lymph node with low USPIO signal decay and bright signal

\section{Discussion}

This work demonstrates the technical feasibility of USPIO-enhanced pelvic MRI at 7-T and shows its potential for diagnosing small lymph node metastases in intensity is annotated with a white arrow and was marked as "without USPIO signal decay." Note particularly the better visibility and delineation of the pathological lymph node and other structures within the mesorectal area at 7-T

patients with rectal cancer and prostate cancer. Our approach mitigates many of the technical challenges associated with 7-T body imaging, enabling large FOV USPIOenhanced imaging of the pelvis without any RF-related signal voids.
Fig. 4 Coronal (a-c) and transversal (d) zoomed computed $\mathrm{TE}=8 \mathrm{~ms}$ images at $7-\mathrm{T}$ of the four patients not depicted in Figs. 2 and 3. a Patient 2 (male, 52 years old, prostate cancer). $\mathbf{b}$ Patient 3 (male, 62 years old, prostate cancer). c Patient 5 (female, 64 years old, rectal cancer). d Patient 6 (female, 55 years old, rectal cancer). The images indicate a lymph node and its short axis that was marked as lymph node without USPIO signal decay by a radiologist (white arrow). Note how these lymph nodes without USPIO signal decay, although small, are clearly visible due to bright signal and can be delineated well from other structures. The node identified in $\mathbf{d}$ shows signal heterogeneity, which could indicate partial USPIO signal decay within this node
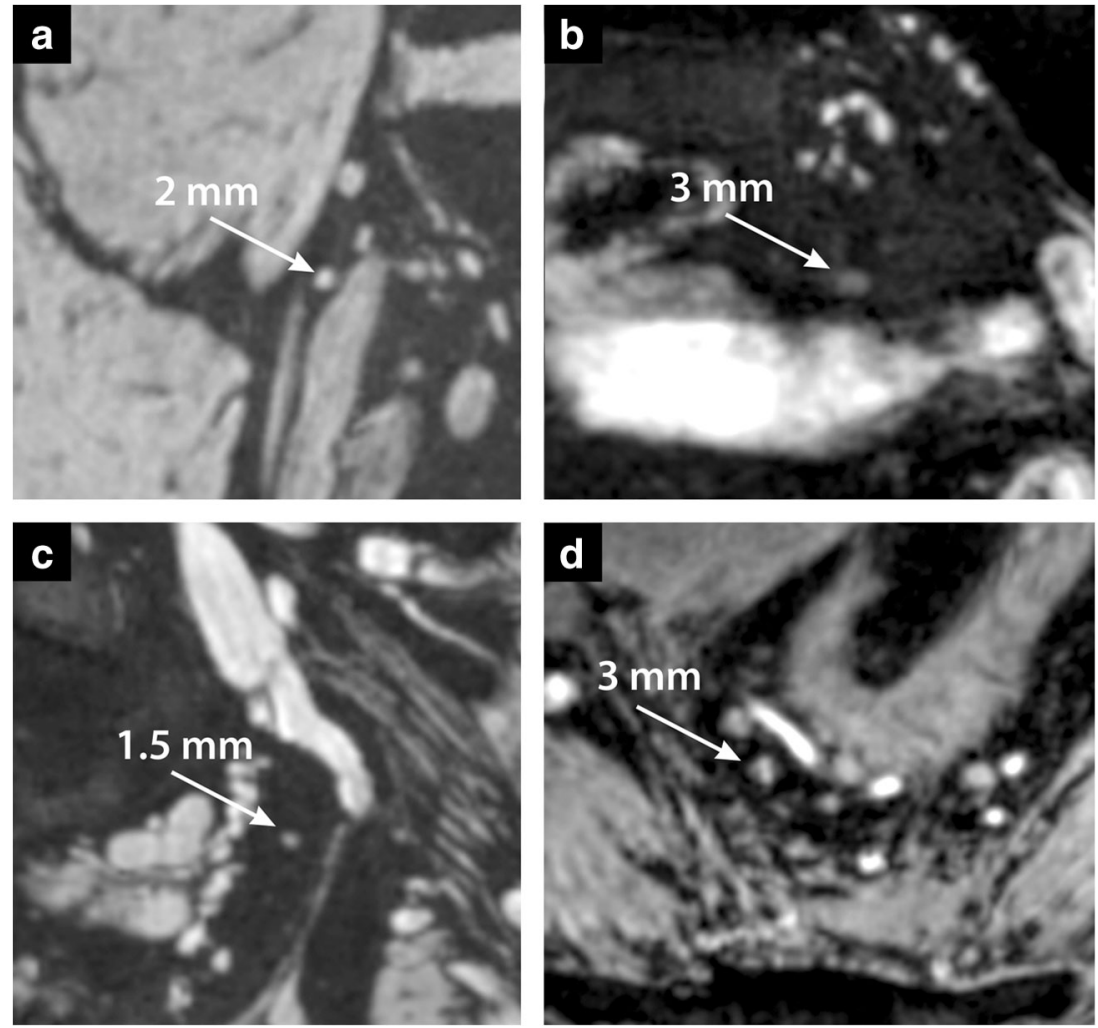
Water-selective mGRE imaging lends itself particularly well for ultrahigh field, as it exploits the $\mathrm{T}_{2}{ }^{*}$ effect of USPIOs and can be performed at low FAs to reduce specific absorption rate (SAR) issues. It can be performed reliably using the TIAMO technique and can already compete with our clinical protocol at 3-T. It provides a higher spatial resolution which may allow the assessment of USPIO uptake in normal-sized lymph nodes down to $1.5 \mathrm{~mm}$ in short-axis diameter or the evaluation of partial uptake within a lymph node.

The B1TIAMO $\mathrm{B}_{1}{ }^{+}$mapping technique enables patientspecific flip angle calibration, such that a similar SNR and contrast are obtained in all patients. It shows relatively homogenous root-sum-of-squares FA distributions.

A general observation of GRE imaging at 7-T is the shorter overall $\mathrm{T}_{2}{ }^{*}$. This is compensated by a shorter $\mathrm{T}_{2}{ }^{*}$ of lymph nodes with USPIO uptake at 7-T and it may even lead to a contrast advantage at higher field strengths. Because this $\mathrm{T}_{2}{ }^{*}$ effect is field strength-dependent, the TE should be adjusted as well to provide optimal distinction between normal and suspicious lymph nodes at 7-T. Current literature is, however, inconclusive about the exact TE that should be used for optimal $\mathrm{T}_{2}{ }^{*}$-weighting, reporting values in the range of $11-27 \mathrm{~ms}$ at 1.5-T [5, 28-30]. Furthermore, these reported values often correspond to effective TEs, which are obtained by the sum of squares combination of several images with different TEs, resulting in ambiguous $\mathrm{T}_{2}{ }^{*}$ contrast. We therefore used computed TE imaging [19], in which a voxel-wise exponential fit is performed over the TEs, pooling information from all images without losing the $\mathrm{T}_{2}{ }^{*}$ contrast information. This approach allows calculation of images at arbitrary TE, so that an optimal computed TE for detecting lymph node metastases can be assessed retrospectively (in validation studies).

As the acquisition time of the current GRE imaging protocol is about $9 \mathrm{~min}$, bowel motion can cause motion artifacts despite administration of butylscopolamine, rendering mesenteric lymph nodes difficult to assess. Fortunately, this did not appear for iliac or mesorectal lymph nodes, particularly when combined with butylscopolamine and bowel preparation [31]. Lymph nodes in prostate and rectal cancer were therefore well visible.

7-T MRI offers advantages in SNR and the susceptibility to USPIOs, which can be used to increase spatial resolution and sensitivity to USPIO uptake compared with current clinical 3T MRI. Its main downside at this time is its limited availability and absence of a standard RF body coil configuration and standard body imaging methods.

This work shows preliminary observations of the use of USPIO-enhanced MRI at 7-T in a small number of patients. Although USPIO-enhanced MRI was validated for the detection of pelvic lymph nodes in previous studies [5], there is not much literature on its validity in the small lymph nodes that are visualized with high-resolution 3D MRI. The patients in this study, particularly those with prostate cancer, show a large number of small lymph nodes without USPIO signal decay. A reason for this large number of nodes could be our selection of prostate cancer patients with a high number of suspicious lymph nodes based on a clinical USPIO-enhanced MRI scan. Whether all these lymph nodes were indeed metastatic could not be validated in this work: no systematic follow-up or nodeto-node pathological data was available. Although lymph nodes with USPIO signal decay were found in all patients, we cannot be sure that our method is sensitive enough to visualize small quantities of USPIO uptake robustly. Moreover, as is known for inguinal lymph nodes [32], there could be regional differences in lymph node USPIO uptake. Larger patient studies with standardized radiological evaluation are therefore necessary to demonstrate the clinical feasibility of USPIOenhanced MRI at 7-T for detecting lymph node metastases, particularly in normal-sized lymph nodes. To perform a validation on lymph nodes of this size requires a very systematic and meticulous correlation between imaging and pathology in a relatively large group of patients and is therefore not within the scope of this technical note. Another possibility would be to compare the results of USPIO-enhanced 7-T MRI with other imaging methods such as PET-CT or diffusion-weighted imaging (DWI). These methods, however, lose sensitivity for smaller lymph node metastases [33] and whole-pelvis DWI at 7-T is technically very challenging.

In conclusion, 3D large field-of-view lymph node MR imaging at 7-T is technically feasible and permits assessing USPIO signal decay in normal-sized lymph nodes throughout the lower abdomen in a patient suffering from primary pelvic cancers. Its clinical effectiveness needs to be evaluated in further clinical studies.

Acknowledgments Dutch Cancer Society: KUN 2014-6624

Funding This study has received funding by the Dutch Cancer Society KUN 2014-6624.

\section{Compliance with ethical standards}

Guarantor The scientific guarantor of this publication is Tom W.J. Scheenen, $\mathrm{PhD}$.

Conflict of interest The authors of this manuscript declare no relationships with any companies, whose products or services may be related to the subject matter of the article.

Statistics and biometry No complex statistical methods were necessary for this paper. 
nformed consent Written informed consent was obtained from all subjects (patients) in this study.

Ethical approval Institutional Review Board approval was obtained.

\section{Methodology \\ - Performed at one institution}

Open Access This article is distributed under the terms of the Creative Commons Attribution 4.0 International License (http:// creativecommons.org/licenses/by/4.0/), which permits unrestricted use, distribution, and reproduction in any medium, provided you give appropriate credit to the original author(s) and the source, provide a link to the Creative Commons license, and indicate if changes were made.

\section{References}

1. McMahon CJ, Rofsky NM, Pedrosa I (2010) Lymphatic metastases from pelvic tumors: anatomic classification, characterization, and staging. Radiology 254:31-46

2. Musch M, Klevecka V, Roggenbuck U, Kroepfl D (2008) Complications of pelvic lymphadenectomy in 1,380 patients undergoing radical retropubic prostatectomy between 1993 and 2006. J Urol 179:923-929

3. Wu L, Cao Y, Liao C, Huang J, Gao F (2011) Diagnostic performance of USPIO-enhanced MRI for lymph-node metastases in different body regions: a meta-analysis. Eur J Radiol 80:582-589

4. Harisinghani MG, Barentsz J, Hahn PF et al (2003) Noninvasive detection of clinically occult lymph-node metastases in prostate cancer. N Engl J Med 348:2491-2499

5. Heesakkers RA, Hövels AM, Jager GJ et al (2008) MRI with a lymph-node-specific contrast agent as an alternative to $\mathrm{CT}$ scan and lymph-node dissection in patients with prostate cancer: a prospective multicohort study. Lancet Oncol 9:850-856

6. Bellin MF, Roy C, Kinkel K et al (1998) Lymph node metastases: safety and effectiveness of MR imaging with ultrasmall superparamagnetic iron oxide particles-initial clinical experience. Radiology 207:799-808

7. Anzai Y, Piccoli CW, Outwater EK et al (2003) Evaluation of neck and body metastases to nodes with ferumoxtran 10-enhanced MR imaging: phase III safety and efficacy study. Radiology 228:777-788

8. Daldrup-Link HE (2017) Ten things you might not know about iron oxide nanoparticles. Radiology 284:616-629

9. Fortuin AS, Brüggemann R, van der Linden J et al (2018) Ultrasmall superparamagnetic iron oxides for metastatic lymph node detection: back on the block. Wiley Interdiscip Rev Nanomed Nanobiotechnol 10:e1471

10. Thoeny HC, Triantafyllou M, Birkhaeuser FD et al (2009) Combined ultrasmall superparamagnetic particles of iron oxideenhanced and diffusion-weighted magnetic resonance imaging reliably detect pelvic lymph node metastases in normal-sized nodes of bladder and prostate cancer patients. Eur Urol 55:761-769

11. Triantafyllou M, Studer UE, Birkhäuser FD et al (2013) Ultrasmall superparamagnetic particles of iron oxide allow for the detection of metastases in normal sized pelvic lymph nodes of patients with bladder and/or prostate cancer. Eur J Cancer 49:616-624
12. Heesakkers RA, Fütterer JJ, Hovels AM et al (2006) Prostate cancer evaluated with ferumoxtran-10-enhanced T2*-weighted MR imaging at 1.5 and 3.0 T: early experience. Radiology 239:481-487

13. Kinner S, Maderwald S, Albert J et al (2013) Discrimination of benign and malignant lymph nodes at $7.0 \mathrm{~T}$ compared to $1.5 \mathrm{~T}$ magnetic resonance imaging using ultrasmall particles of iron oxide: a feasibility preclinical study. Acad Radiol 20:1604-1609

14. Christoforidis GA, Yang M, Kontzialis MS et al (2009) High resolution ultra high field magnetic resonance imaging of glioma microvascularity and hypoxia using ultra-small particles of iron oxide. Invest Radiol 44:375-383

15. Birkhäuser FD, Studer UE, Froehlich JM et al (2013) Combined ultrasmall superparamagnetic particles of iron oxide-enhanced and diffusion-weighted magnetic resonance imaging facilitates detection of metastases in normal-sized pelvic lymph nodes of patients with bladder and prostate cancer. Eur Urol 64:953-960

16. Pohmann R, Speck O, Scheffler K (2016) Signal-to-noise ratio and MR tissue parameters in human brain imaging at 3, 7, and 9.4 tesla using current receive coil arrays. Magn Reson Med 75:801-809

17. Brisset JC, Desestret V, Marcellino S et al (2010) Quantitative effects of cell internalization of two types of ultrasmall superparamagnetic iron oxide nanoparticles at $4.7 \mathrm{~T}$ and $7 \mathrm{~T}$. Eur Radiol 20:275-285

18. Kim SH, Oh SN, Choi HS et al (2016) USPIO enhanced lymph node MRI using 3D multi-echo GRE in a rabbit model. Contrast Media Mol Imaging 11:544-549

19. Philips BWJ, Fortuin AS, Orzada S, Scheenen TWJ, Maas MC (2017) High resolution MR imaging of pelvic lymph nodes at 7 Tesla. Magn Reson Med 78:1020-1028

20. Ugurbil K (2014) Magnetic resonance imaging at ultrahigh fields. IEEE Trans Biomed Eng 61:1364-1379

21. Orzada S, Maderwald S, Poser BA, Bitz AK, Quick HH, Ladd ME (2010) RF excitation using time interleaved acquisition of modes (TIAMO) to address B1 inhomogeneity in high-field MRI. Magn Reson Med 64:327-333

22. Orzada S, Quick HH, Ladd ME et al (2009) A flexible 8-channel transmit/receive body coil for $7 \mathrm{~T}$ human imaging. Proc Intl Soc Mag Reson Med 17:2999

23. Rietsch SHG, Orzada S, Maderwald S et al (2018) 7T ultra-high field body MR imaging with an 8-channel transmit/32-channel receive radiofrequency coil array. Med Phys 45:2978-2990

24. Rietsch SH, Quick HH, Orzada S (2015) Impact of different meander sizes on the RF transmit performance and coupling of microstrip line elements at 7 T. Med Phys 42:4542-4552

25. Orzada S, Maderwald S, Poser BA et al (2012) Time-interleaved acquisition of modes: an analysis of SAR and image contrast implications. Magn Reson Med 67:1033-1041

26. Brunheim S, Gratz M, Johst S et al (2018) Fast and accurate multichannel B1+ mapping based on the TIAMO technique for 7T UHF body MRI. Magn Reson Med 79:2652-2664

27. Veraart J, Sijbers J, Sunaert S, Leemans A, Jeurissen B (2013) Weighted linear least squares estimation of diffusion MRI parameters: strengths, limitations, and pitfalls. Neuroimage 81:335-346

28. Rockall AG, Sohaib SA, Harisinghani MG et al (2005) Diagnostic performance of nanoparticle-enhanced magnetic resonance imaging in the diagnosis of lymph node metastases in patients with endometrial and cervical cancer. J Clin Oncol 23:2813-2821

29. Nguyen BC, Stanford W, Thompson BH et al (1999) Multicenter clinical trial of ultrasmall superparamagnetic iron oxide in the evaluation of mediastinal lymph nodes in patients with primary lung carcinoma. J Magn Reson Imaging 10:468-473 
30. Harisinghani MG, Saini S, Weissleder R et al (1999) MR lymphangiography using ultrasmall superparamagnetic iron oxide in patients with primary abdominal and pelvic malignancies: radiographic-pathologic correlation. AJR Am J Roentgenol 172: $1347-1351$

31. Gutzeit A, Binkert CA, Koh DM et al (2012) Evaluation of the antiperistaltic effect of glucagon and hyoscine on the small bowel: comparison of intravenous and intramuscular drug administration. Eur Radiol 22:1186-1194

32. Froehlich JM, Triantafyllou M, Fleischmann A, Vermathen P, Thalmann GN, Thoeny HC (2012) Does quantification of USPIO uptake-related signal loss allow differentiation of benign and malignant normal-sized pelvic lymph nodes? Contrast Media Mol Imaging 7(3):346-355

33. Herlemann A, Wenter V, Kretschmer A et al (2016) ${ }^{68}$ Ga-PSMA positron emission tomography/computed tomography provides accurate staging of lymph node regions prior to lymph node dissection in patients with prostate cancer. Eur Urol 70(4):553-557

Publisher's note Springer Nature remains neutral with regard to jurisdictional claims in published maps and institutional affiliations. 